Document downloaded from:

http://hdl.handle.net/10251/52779

This paper must be cited as:

Soriano Olivares, J.; Arregui De La Cruz, F.; Espert Alemany, VB.; García-Serra García, J. (2014). Hydraulic transient in residential buildings with a direct pump connection. Urban Water Journal. 2014:1-13. doi:10.1080/1573062X.2014.989860.

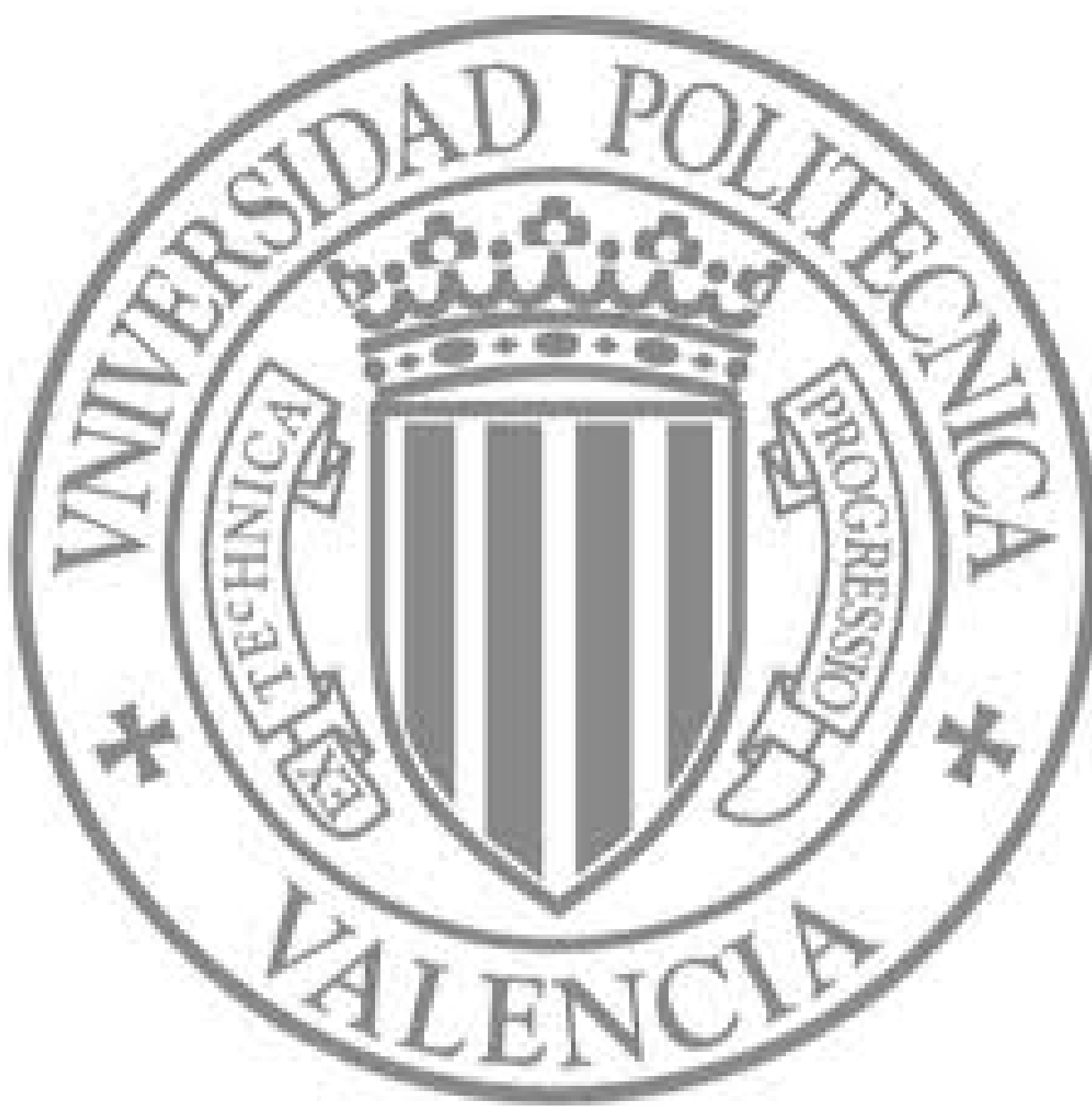

The final publication is available at

http://dx.doi.org/10.1080/1573062X.2014.989860

Copyright Taylor \& Francis 


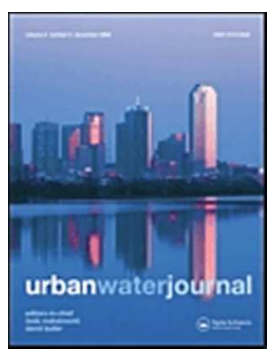

\section{Hydraulic transient in residential buildings with a direct pump connection}

\begin{tabular}{|r|l|}
\hline Journal: & Urban Water Journal \\
\hline Manuscript ID: & Draft \\
\hline Manuscript Type: & Full Paper \\
\hline Date Submitted by the Author: & n/a \\
\hline Complete List of Authors: & $\begin{array}{l}\text { Soriano, Javier; Universitat Politécnica de Valencia, Departamento de } \\
\text { Ingeniería Hidráulica y Medio Ambiente } \\
\text { Arregui, F.; Universitat Politécnica de Valencia, Departamento de } \\
\text { Ingeniería Hidráulica y Medio Ambiente } \\
\text { Espert, V.; Universitat Politécnica de Valencia, Departamento de Ingeniería } \\
\text { Hidráulica y Medio Ambiente } \\
\text { García-Serra, Jorge; Universitat Politécnica de Valencia, Departamento de } \\
\text { Ingeniería Hidráulica y Medio Ambiente }\end{array}$ \\
\hline Keywords: & $\begin{array}{l}\text { Water Distribution Modelling, Water Supply, Urban water systems } \\
\text { interactions, Hydraulic transient, Pumping systems, Water hammer }\end{array}$ \\
\hline
\end{tabular}

\section{SCHOLARONE}

Manuscripts 


\section{Hydraulic transient in residential buildings with a direct pump connection}

Soriano J*, Arregui F., Espert V.B. and García-Serra, J.

Department of Hydraulic and Environmental Engineering

ITA. Universitat Politècnica de València

Camino de Vera s/n, 46022, Valencia, Spain.

*Email: jasool@ita.upv.es. Tel.:+3496387 9898. Fax: +34963879899

This work was supported by the The Ministerio de Educación y Ciencia of the Spanish Government under Grant N ${ }^{\circ}$ CGL2005-03666.

\section{1}

URL: http:/mc.manuscriptcentral.com/nurw Email: urbanwater@exeter.ac.uk 


\section{Hydraulic transient in residential buildings with a direct pump connection}

This paper consists of an experimental and numerical study into transient behavior in a residential building. The analyzed effects are originated by centrifugal pumps during their starting and shutdown period when a direct supply system is in place. In this system, fixedspeed pumps are connected to the service pipe without an atmospheric tank, in order to avoid the water quality problems associated. Direct supply systems magnify the transient effect inside the installation and place higher demands on the water main.

The properties of such an installation were analyzed using a hydraulic model in order to detect the most unfavorable scenario. The results were compared with experimental data obtained directly from pressure measurements. In general, the results show that slow changes in flow rate are recommended, and also that reducing the distance between the pump and the service pipe, or using large diameter pipes, can reduce the pressure surge.

Keywords: Hydraulic transient; water hammer; direct supply; water plumbing system; pump station. 


\section{INTRODUCTION}

Urban population growth is significantly increasing the demand on water distribution systems. Despite this growing need for water, the required infrastructure transformation has not always taken place at an adequate rate in many countries, leading to insufficient water supply capacity and poor service quality (Kirmeyer et al. 1994; Clark et al. 2002). In those cities where water supply capacity cannot meet the instantaneous water demand during peak hours, users are forced to install private storage tanks that can provide them with the water they need during the hours of maximum consumption. Furthermore, even in systems with continuous supply, the instantaneous water demand of many users may be too large for the transportation capacity of the network. Therefore, a private storage tank that can guarantee a reserve of water and the instantaneous consumption flow rate at any time becomes indispensable for large users. In addition, local regulations may even require atmospheric tanks upstream pumping systems to be installed inside the customer's building in order to minimize the magnitude of the flow rates extracted from the network.

In such cases, water consumption of users owning a private storage tank is decoupled from the water flows extracted from the network. In these installations, pumps are not connected directly to the service pipe and large instantaneous water demands are not extracted from the network. An additional advantage associated with tanks is that refilling may take place at any time and at lower flow rates without overloading the network.

\section{Indirect supply system problems}

Nevertheless, the utilization of atmospheric storage tanks is not always a suitable technical solution when used in residential buildings. The main disadvantage is probably that of water quality degradation. In the first place, the storage tank itself contributes to the deterioration in the water quality, as maintenance or periodic cleaning of storage tanks is often not conducted adequately, if it is conducted at all. As these elements are installed inside the users' own facilities and owned by them, water companies cannot undertake the required maintenance and cleaning activities. In other words, water companies have no control over the quality of the water after it reaches the tanks. 
Additionally, an extended period of storage in the tank causes significant losses in chlorine residual (Rossman et al. 1994; Hua et al. 1999; Woolschlager et al. 2005). Unfortunately, increasing the chlorine dosing rate to preserve concentration values may lead to an increase in disinfection byproducts (DBP) such as trihalomethanes (THMs) or haloacetic acids (HAAs) through contact with organic compounds (Nieuwenhuijsen et al. 2000; Levesque et al. 2006; Courtis et al. 2009). Most of these are considered to be potentially carcinogenic (Cantor et al. 1998). This question is of particular interest in Mediterranean regions with very high concentrations of organic matter, where THM levels could be as high as $90 \mu \mathrm{g} / \mathrm{l}$ (Villanueva et al. 2003). Furthermore, consideration should be given to other quality problems, such as the variation of $\mathrm{pH}$ levels and the growth of algae and other biological organisms. Extended storage periods, sediments, biofilms, the presence of nutrients or temperatures of around $25^{\circ} \mathrm{C}$ provide favorable conditions for these negative effects (Mohamed and Gad 2011). Finally, the possibility of dirt or different types of living organisms falling into the tank cannot be ignored, especially when storage tanks are installed underground or are outside of the building.

The utilization of storage tanks is also associated with energy efficiency problems and carbon footprint (Basupi et al. 2014). Water depressurization always involves a waste of energy, as the fluid elastic energy is unnecessarily dissipated. The amount of energy required to supply customers with the desired pressure and flow is significantly larger when water is taken from an atmospheric storage tank than when it is taken from a pressurized network. Some authors estimate energy losses of up to $30 \%$ when depressurizing water into storage tanks (Wilo AG 2007; Cobacho et al. 2008). Savings when customers do not use these tanks not only come from improving energy efficiency but also from the lower cost of the pumping equipment required to supply the customers.

An additional drawback to using private storage tanks consists of the consequent reduction in the hydraulic efficiency of the network. Water losses in these elements are frequent (Tamari and Ploquet 2012), as level control valves installed at the inlet are not completely watertight some time after installation (Rizzo and Cilia 2005; Charalambous et al. 2007; Criminisi et al. 2009), particularly

4 URL: http:/mc.manuscriptcentral.com/nurw Email: urbanwater@exeter.ac.uk 
in those systems with poorer water quality. Also, metering efficiency significantly decreases due to the low flows at which the storage tanks are usually refilled.

As a result, and given that water quality is a major concern for residential customers, many water companies are searching for technical solutions that could allow the removal of storage tanks from the facilities of domestic users. It seems clear that individual storage tanks can be removed from many systems without significant problems, as instantaneous flow rates will not substantially increase in the part of the network owned by the water company. However, the removal of collective storage tanks poses a risk of damage to the network if proper precautions are not taken. In such cases, the required consumption flow rates of the installation may be too large for the capacity of the network. A typical example of such installations are those found in residential buildings in which booster pumping systems usually withdraw water from storage tanks installed at ground level: this type of facility is known as an indirect supply system. If the storage tank is removed, the pumps would withdraw the water directly from the network (direct supply system).

\section{Direct supply system problems}

Despite the clear advantages of removing private storage tanks, the use of a direct supply system can have two negative effects. Firstly, during the steady state operation of the pump, the flow rate withdrawn from the network may be too great for the capacity of the latter, decreasing water pressure in the surrounding area to unacceptable levels. Secondly, during start-up and shut-down operations, pressure surges may produce under and over-pressures of a large magnitude that can, in the best-case scenario, degrade network infrastructure in the long term, and in the worst case, produce an immediate failure of the public mains.

Historically, despite their primary importance, the effects of water transients have been thought to be of most interest to the designers and operators of large-scale projects. The study of water transients within urban water supply networks has been neglected in the technical literature and significant references are scarce. This can be partially explained by the fact that while the steady state behavior of the network can be studied at almost no cost with free-to-use software, the study of water

5 URL: http:/mc.manuscriptcentral.com/nurw Email: urbanwater@exeter.ac.uk 
transients is a much more complex subject which requires specific and expensive analytical software. In most cases, the analysis of water transients is not justified for a relatively small single customer.

This paper explores the possibility of removing collective atmospheric tanks in residential buildings and connecting booster pumping systems directly to the network. As transient effects during the start-up of the pumps have been proven to be significantly more severe than under steady state conditions, the research focuses on an analysis of the under-pressures generated during the startup of the pumps.

\section{MATHEMATICAL MODEL}

\section{Model description}

In order to understand the transient effects associated with the direct connection of the pumping stations of residential buildings to the public water network, a general hydraulic model was developed. The software package used for this purpose was ALLIEVI, which implements the method of characteristics (MOC, Wylie and Streeter 1993). ALLIEVI, which is a freeware program developed at the Universitat Politècnica de València, allows for the hydraulic simulation of complex water installations, such as those which are typical of residential buildings.

For the purpose of this study, a comprehensive numerical analysis was conducted by changing the value of the physical variables used in the mathematical model, including the properties of the pipes, the installation layout in the building and the pumping station characteristics. By doing so, it was possible to determine their influence on the magnitude of the pressure transient and to propose a protection strategy for the public water network and the water installation inside the building. Among all the parameters considered, two of them should be of primary concern for the designer: the pumping station flow rate capacity and the relationship between the diameter of the service pipe and the public main.

In order to obtain accurate results, the analysis of the water transient cannot be simulated without considering the surrounding water network. Consequently, some simplifications need to be conducted in order to maintain simulation times below reasonable figures. A simplified model which

6

URL: http:/mc.manuscriptcentral.com/nurw Email: urbanwater@exeter.ac.uk 
considers the most important parameters is proposed for the study. A schematic of this simplified model is shown in Fig. 1. It includes four reservoirs (T1 - T4) that play the role of the larger diameter mains belonging to the water supply company. These tanks are interconnected using pipes of different diameters, lengths and materials. By changing the water level of the tanks, the flow rate of water circulating through each pipe can be easily modified and adjusted to the desired values.

The water supply installation within the residential building is connected to the main by means of a service pipe at the connection point (Fig. 1). The water plumbing system includes a pumping station that can be equipped with several parallel pumps and other elements.

The main properties of all the elements considered in the model can be easily changed in the simulation software. More specifically, with regard to the pumping station, the performance curves of the pumps can be defined by using the operating points provided by the manufacturers or by using Suter universal curves. Other properties such as the inertia of the pump, the starting time, or the nominal rotational speed are also editable.

Water demand inside the building is modeled using a throttle control valve $(\mathrm{V})$ and a reservoir (T5) with a constant level. By modifying the valve properties, it is possible to simulate water demand variability through time and adjust it to the real measured values. Additionally, by modifying the level of the tank $\mathrm{T} 5$ it is possible to set the pump pressure output to the required values.

\section{Field validation of the mathematical model}

In order to validate the mathematical model and the proposed simplifications, a residential building was monitored during 6 months. The building studied was located in the city of Seville, Spain (Soriano, 2012), and it consisted of 18 residential units on 6 floors. Each apartment contained 11 appliances distributed across two bathrooms and a kitchen. During the monitoring period, the average occupation was approximately 2.6 persons per household. The maximum instantaneous flow per residence unit, considering the simultaneous use of different appliances, was close to $0.5 \mathrm{l} / \mathrm{s}$. The maximum consumption flow rate measured for the whole building during the monitoring period was approximately $1.8 \mathrm{l} / \mathrm{s}$. However, this consumption peak was only detected for around 15 seconds on 
specific occasions. The public mains were made of ductile cast iron and had an internal diameter of $150 \mathrm{~mm}$. The total length of the service pipe was approximately $20 \mathrm{~m}$. The public service pipe was made of polyethylene PE100 having a nominal pressure of 1MPa, an internal diameter of $55.4 \mathrm{~mm}$ and a length of $5 \mathrm{~m}$. The private service pipe was made of galvanized steel, with an internal diameter of $53.1 \mathrm{~mm}$ and a length of $18 \mathrm{~m}$.

During certain monitoring periods, the building was equipped with two pressure transducers - PS1 and PS2 in Fig. 2 - and the corresponding data loggers to register pressure with a time interval of $0.1 \mathrm{~s}$. At the connection point (PS1), water pressure was almost invariable throughout the day at around $320 \mathrm{kPa}$. Also, all individual water meters and the building water meter were equipped with pulse emitters connected to data loggers. Water consumption in the individual apartments was recorded with a resolution of 0.11 and $0.02 \mathrm{~s}$ while for the bulk meter the available volume resolution of the pulse emitter was 11 .

In accordance with Spanish building regulations, the building was initially equipped with an indirect supply system in which the pumps were connected to an atmospheric tank installed in the basement. Under this configuration all homes are supplied with a fixed-speed pump connected to the tank. Before starting the monitoring period, however, the water installation of the building was modified in order to analyze the effect on the network of different types of pumping stations. The first set-up tested was that with the original fixed-speed pumps installed in the building. The second configuration examined used the brand new variable speed-pumps installed specifically for the study. In either case, for the research conducted, the pumps were connected directly to the water network and not to the atmospheric tank. As shown in Fig. 2, it was possible to switch between both configurations by simply opening and closing the necessary valves.

The technical characteristics of the variable-speed pumping station were selected so that they would be as similar as possible to the original fixed-speed pumping station installed in the building. Both operated with two centrifugal pumps in parallel, each pump having a nominal power of approximately $2 \mathrm{~kW}$, an individual flow capacity of $2.5 \mathrm{l} / \mathrm{s}$ and a pressure of $550 \mathrm{kPa}$ at the optimal

8 URL: http:/mc.manuscriptcentral.com/nurw Email: urbanwater@exeter.ac.uk 
operating point. Under normal working conditions, only one pump was started while the other was kept as a reserve pump.

\section{Measured effects with direct supply and fixed-speed pumps}

To convert the installation into a direct supply system with fixed-speed pumps, valves V2 was opened while valves V1 and V3 were closed (Error! Reference source not found.). The pressure switch controlling the pumps was adjusted so that the minimum downstream pressure at the pump was approximately $550 \mathrm{kPa}$. Therefore, the flow rate withdrawn from the water network, once the pump had started and reached the steady state, was slightly larger than $2.5 \mathrm{l} / \mathrm{s}$. This is due to the fact that the upstream pressure at the pump inlet changed from almost zero (indirect supply) to a pressure slightly lower than the network pressure (direct supply), modifying the operating point of the pump.

Such rapid change of the water flow rate circulating through the service pipe, typically in less than a second, poses a risk of damage to the public water supply system or other private installations in the surrounding area. A noticeable drop in pressure takes place during these first instants of the starting, especially at the pipe sections closest to the pumps. On the other hand, under-pressures at the connection point between the public main and the service pipe are considerably reduced in magnitude due to the mitigating effect of a large diameter pipeline.

According to the measurements taken at the specific installation monitored, the pressure head drop during the transient, $\Delta H_{T}$, was close to $15-20 \mathrm{~m}$ (Fig. 3-a). However, the amplitude of the pressure oscillation may strongly differ from one installation to another and this observation cannot be easily generalized. The most critical point is during the first seconds after starting the pumps, at the suction section. Typically, this initial under-pressure lasts for a short period of approximately 0.5 $\mathrm{s}$, although its exact duration depends on the length of the service pipe and the wave celerity. Its magnitude is strongly related to the operating point of the pumps and the time required for the nominal speed to be reached. This last parameter is not usually provided by pump manufacturers and is quite difficult to measure in the field. 
While the most severe under-pressure is found on the suction side of the pumps (transducer PS2), the pressure oscillation is significantly smaller at a point closer to the connection between the service pipe and the main pipeline (transducer PS1). The mitigation of the pressure surge is facilitated by the public main that maintains the pressure to acceptable levels, acting in a similar fashion to a constant level reservoir. However, under some circumstances, this mitigation effect may not be enough depending on the diameter of the public main and the flow capacity of the pumps. The fact that damage to the public infrastructure as a consequence of these working cycles may occur in the medium or long term should also be taken into account. Small but frequent pressure surges can cause an unpredictable failure of the public mains and can have the same effect as larger surges.

Another negative effect that should be taken into consideration when connecting the pumps directly to the water network is the pressure drop under steady state conditions that takes place after the initial pressure transient. Its magnitude depends on the transportation capacity of the public main and the service pipe with respect to the design flow rate of the pumps. In any case, this effect can be easily predicted using standard steady state simulation software such as EPANET.

Finally, it should be noted that a pump shutdown may also cause pressure surges. However, the magnitude of this transient is in most cases negligible due to the protection provided by the pressure vessel installed downstream of the pumps. For this reason, the present study only focused on the analysis of the pressure transient generated during the start-up of a pump.

\section{Measured effects with direct supply and variable-speed pumps}

Switching from fixed-speed pumps to variable-speed pumps only required opening valve V3 and closing valves V1 and V2 (Fig. 2). Quality of service to the residents of the building, perceived as the amount of water flowing out from their faucets, was maintained with respect to fixed-speed pumps by setting the target downstream pressure at the pump to $500 \mathrm{kPa}$.

As the start-up ramp is known to be a critical parameter in the magnitude of the pressure surge, this parameter was set to $3 \mathrm{~s}$ in the controller of the variable-speed drive. By doing this, the frequency response of the pump to changes in water consumption is relatively slow. However, if the pressure

10

URL: http:/mc.manuscriptcentral.com/nurw Email: urbanwater@exeter.ac.uk 
vessel installed downstream the pump station is of the appropriate size, this slow response is not perceived by customers at any time. Also, an additional factor to be considered when using variablespeed pumps is that downstream pressure is not completely stable. Some minor oscillations are always observed (Fig. 3-b). The amplitude of the pressure ripple observed depends on the PID settings of the controller, the pressure vessel properties and the pressure oscillations of the network. In the installation monitored, measurements showed that pressure surges were considerably reduced in comparison with the situation in which equivalent fixed-speed pumps are used. Under-pressure reduction can be quantified as a $90 \%$ of the measured value when fixed-speed pumps were used. The reduction was observed for both under-pressure and over-pressure caused by starting/stopping the pumps, leading to an almost constant pressure at any point of the installation at any time. Under these circumstances, a specific analysis for the water transient has proven unimportant. For the protection of the installation and the public water network, only the steady state conditions should be taken into consideration.

\section{Validation of the simulation results}

The field validation of the numerical model was an important stage in this study. For this purpose, field tests and computational simulations were conducted under the same conditions in order to validate the simplified mathematical model. For comparison purposes, equivalent pressure and flow monitoring points were defined in both the hydraulic model and the field installation. More specifically, points A and B (Fig. 1) corresponded to PS1 and PS2 (Fig. 2) respectively. Also, the properties of the elements considered in the mathematical model corresponded as closely as possible to the physical properties of the water installation monitored. This includes pipe lengths, internal diameters and materials, pump characteristics, pressure vessel dimensions and properties, etc. However, as the physical model was a real water installation rather one in a laboratory, it was impossible to reproduce in every detail all of the physical variables that could affect the pressure surge magnitude. Some parameters, such as the fluid-structure interaction, bends, water meters, and check-valves, were not taken into account in the mathematical model.

11 URL: http:/mc.manuscriptcentral.com/nurw Email: urbanwater@exeter.ac.uk 
Fig. 3-a also shows a comparison between simulated and measured data for a typical start-up and shutdown of the fixed-speed pumps installed in the building. The pressure readings at the PS1 and PS2 locations are plotted. Simulated results obtained with ALLIEVI agree relatively well for the first pressure drop (during the start-up of the pump) and the first pressure peak. During the initial instants of the transient, when the pump is switched on, the course of the simulated pressure surge corresponds in phase and magnitude with the measured pressure. However, the comparison clearly indicates that the discrepancies between the numerical and simulated results are significant after the first cycle. In a real installation, pressure fluctuations are rapidly attenuated and the pressure wave is delayed in time. This effect - in terms of the shape, amplitude and phase of the pressure wave - has been thoroughly studied by different researchers (Covas et al. 2004; Bergant 2008), and it is commonly assumed that is associated with fluid-structure interaction and model simplifications (for instance, pipe-wall viscoelasticity, friction factor, etc.). In any case, this is not a problem for this study, as the critical results always appear during the first pressure oscillation and the results obtained from the numerical model are on the safer side (the predicted pressure surge was greater than the real one).

\section{SENSITIVITY ANALYSIS}

In order to evaluate the influence of the different variables considered, a sensitivity analysis was also conducted using ALLIEVI. In this analysis, the actual water hammer originated by the pump was defined as the reference transient. The variables analyzed were the pressure drop observed at the connection between the service pipe and the public water main (PS1) and at the suction pipe of the pumps (PS2). In this analysis, only one parameter of the model was modified while the rest of considered variables were kept invariant. The variables analyzed corresponded to the key properties of the main pipeline - diameter, pressure at the connection point and flow - and the plumbing system properties - starting time of the pump, design flow and head of the pump, diameter and length of the service pipe.

12

URL: http:/mc.manuscriptcentral.com/nurw Email: urbanwater@exeter.ac.uk 
To analyze this variable, the water levels of the tanks (T1 to T4) connected to the public mains were appropriately modified to attain the desired flow while maintaining pressure values at PS1. By changing the water head of one tank it was possible to achieve different flow rates in the mains. Modifying the remaining tank levels allows the pressure to be maintained at the connecting point at the desired value. As expected, Fig. 4-a shows that variations of the flow rate through the public main had no effect on the pressure drop at the connection point when the pump started up.

\section{Diameter of the public main}

When the diameter of the service pipe is modified, the pressure drop at the connection point was subject to significant changes not only during the transient phase but also during the steady state (Fig. 4-b). Smaller diameters originate larger pressure drops. On the other hand, larger diameters reduced the under-pressure magnitude. The under-pressure reduction strongly depends on the size of the service pipe. As shown in this figure, increasing the diameter of the service pipe above a certain value does not significantly reduce under-pressure values.

Pressure drop reductions with larger diameters can be explained in terms of pressure wave transmission and reflection at the connection point. When the transient pressure wave is generated inside the building during the startup of the pump, a negative pressure wave of magnitude $\Delta H$ impinges at the junction. This wave is partially reflected towards the inside of the building with a magnitude of $T \cdot \Delta H$ (where $T$ corresponds to the reflection coefficient) and opposite sign. Likewise, the complementary part of the wave is transmitted to the service pipe. The magnitude of the wave reflected (Eq. 1) can be calculated by means of the reflection coefficient $\mathrm{T}$ which is a function of the diameters of the pipes and the kinematic wave celerity $c$ (Wylie and Streeter 1993). According to this equation, and assuming that wave celerity is the same for all pipes, a service pipe having a diameter three times larger than the connection pipe will reflect $89 \%$ of the wave and $11 \%$ will be transmitted.

However, if the service pipe diameter is four times larger than the connection pipe, wave reflection increases only to a slightly higher figure, $93 \%$.

13 


$$
T=\frac{\frac{A_{W M}}{c_{W M}}-\frac{A_{S P}}{c_{S P}}}{\frac{A_{W M}}{c_{W M}}+\frac{A_{S P}}{c_{S P}}}
$$

where $A_{W M}$ corresponds to the public water main section area and $A_{S P}$ is the service pipe section area.

When a small percentage of the pressure wave is transmitted to the public main there is no need to protect the network against the water transient originated by the pump at start-up or shut-down operations. In such cases, the public main is acting similarly to a tank of constant head that does not allow the pressure wave to travel into the water network.

For the installation monitored, a public main of $150 \mathrm{~mm}$ is enough to provide sufficient protection to the public main pipeline. The transmission coefficient is approximately $11 \%$.

\section{Distance of the building connection to nearby main junctions}

The value of the drop in pressure at the connection point in the building could only be obtained by taking into account the transmitted pressure wave from the inside of the building and the reflections at other customer connections and nearby public main junctions. The fingerprint of these reflections was distinctive to each specific customer connection. The greatest drop in pressure appeared when no reflections of the under-pressure wave were recorded in the contiguous public mains. This is the case when the building connection is at the very end of a pipeline and the closest main junction is located at a distance such that the traveling time of the wave is longer than half the time the pump needs to reach its nominal rotational speed. In such cases, the pressure drop will correspond to the complete pressure wave transmitted to the public main and it can be easily calculated using the Joukowski equation and the increase in velocity at the public main caused by the pump start-up.

In some installations, the pressure wave is partially mitigated by the reflections at the nearby main junctions. The pressure drop at the connection can be roughly estimated by using the Michaud equation between the connection and the closest main junction. A safety coefficient may be also applied when a significant part of the wave is expected to be transmitted at the pipe junction (Eq. 1).

14 
In any case, in complex systems with numerous junctions and customer connections the actual underpressure will always be smaller than the one predicted with Michaud equation.

\section{Pressure head at the connection point}

By changing water levels at tanks $\mathrm{T} 1$ and $\mathrm{T} 2$ it was possible to take into consideration different pressure values at the connection point of the building. Results from the simulations showed that lower pressure values at the connection point produce slightly larger pressure drops. This effect was also observed during the field measurement stage in PS2 (shown in Fig. 4-c).

These results can be explained in terms of how the start-up of the pump is simulated by the software package used in this study. When the pressure head upstream of the pump increases, a smaller head is required in order to provide the same pressure downstream of the pump. As a result, the steady state flow rate (velocity) extracted by the pump will be larger for higher upstream pressures. However, the non-return valve, typically installed downstream of the pump, prevents the circulation of water until the pressure provided by the pump reaches the pressure at the pressure vessel. This means, that for the same starting sequence, the time required to initiate the circulation of water will be longer when the pressure upstream is lower $(0.25 \mathrm{~s}$ for PS1 equal to $330 \mathrm{kPa}$ and $0.35 \mathrm{~s}$ for PS1 equal to $130 \mathrm{kPa}$ ). Since the software assumes a linear increase of the pump rotational speed, larger accelerations of the fluid column are generated when upstream pressure becomes lower. This is true regardless of whether or not the steady state flow velocity is smaller than for higher upstream pressures (Fig. 5).

\section{Starting time of the pump}

The startup time of the pump is defined in the present study as the time taken by the motor to reach its nominal rotational speed. As in other studies, and given the impossibility of employing a simple acceleration law, the analysis of the pump startup is based on the assumption that the rotational speed increases in a linear fashion from zero to the nominal speed (Thorley and Chaudry 1996).

The validation of this critical assumption is extremely difficult. In practice, the startup time cannot be obtained easily in a real installation due to the short duration of the event (sometimes less

15 
than one second) and the high cost of the equipment required for its determination. Consequently, field measurements are rarely obtained except for large motors. As this time depends on the polar moment of inertia for the rotating parts and the difference between available motor torque and load torque, the results obtained for large motors cannot be easily extrapolated to smaller ones.

In any case, this parameter was identified as one of the most critical variables in the pressure surge magnitude. In order to evaluate its influence, simulations with different startup times were conducted. Fig 4-d clearly shows that increasing the startup time reduces under-pressure magnitude, while quick startups generate significant pressure drops. According to the simulation results, the starting time of the pump installed at the validation installation was close to $0.5 \mathrm{~s}$. This figure is similar to the one used in other studies (Zhang et al, 2012).

When the pump is equipped with a variable speed drive it is possible to modify the startup time by controlling the rate at which the rotational speed increases. A variable-speed pump has its rotational speed adjusted in response to the actual demand. This control device ensures that a constant pressure downstream of the pump is maintained at all times independently of the water demand in the installation. When programming the control response, a large number of options are available. As a general rule, selecting a slow response of the controller will always lead to smaller pressure drops during the start-up and shut-down events. On the other hand, selecting configuration parameters for a fast response of the pumps will significantly increase pressure surge magnitude. Therefore, selecting the right configuration for the controller becomes a critical step in order to reduce transient effects caused by centrifugal pumps in buildings.

Even when installing variable speed pumps, an appropriately designed installation will always take into account the effects of the pump when working at a fixed speed. In fact, some regulatory frameworks allow variable speed pumps to operate as fixed speed pumps under certain circumstances, for example during a variable speed drive failure. 


\section{Pump capacity}

If pumps of different capacities are selected, the flow rate actually extracted from the public water main changes depending on the flow capacity of the pump. Hence, a variety of scenarios can be simulated by selecting, from commercial catalogues, pumps of different capacities that could, theoretically, be used in the installation. For this purpose, seven centrifugal pumps were chosen such that the operating point provided a pressure head close to $20 \mathrm{~m}$ when pumping the estimated flow required by the installation. These seven pumps were modeled in ALLIEVI by defining their performance curves (Head-Flow). The pumps are labelled numerically from P0 to P6, where P0 corresponds to the pump used in the reference scenario (which is also actually installed in the building), with the results shown in Fig 4-e. Information of the pressure head at zero flow, $\mathrm{H}_{0}$, is also shown in this figure. The pressure drop magnitude rises in a linear fashion with an increasing flow rate capacity of the pump, while the starting time and other parameters remain constant.

At this stage it is important to mention that large apartment buildings usually incorporate several smaller pumps connected in parallel, rather than a single oversized pump. Such a configuration improves water supply efficiency, as the pump head is maintained within a relatively narrow range and the pumps can operate with a higher average performance. Furthermore, the maximum pressure drop caused by several pumps connected in parallel is lower than the one produced by a single pump with the same flow capacity. The comparison between the operation of one single pump or two parallel pumps is shown in Fig. 6. The reason for this is that the start delay timer, usually integrated in the control system of the pumps, prevents the simultaneous starting of all pumps. Typically the delay is set to 1 or 2 seconds. It was seen that reducing the delay time results in the pressure drop caused by several pumps in parallel tending to match the one produced by a single equivalent pump. Also, the pressure drop originated by the start of a second pump is less than the one caused by the start of the first pump. This can be explained by the fact that the second pump extracts a smaller flow rate than the first pump. Consequently, the pressure drop generated by the velocity change is smaller than when the first pump is started.

17

URL: http:/mc.manuscriptcentral.com/nurw Email: urbanwater@exeter.ac.uk 


\section{Service pipe diameter}

Available commercial diameters of Polyethylene PE-100 were used for the simulation of different service pipes (SP). The internal service pipe diameters studied ranged from 44 to $135 \mathrm{~mm}$. Figure4-f shows that the pressure drop magnitude decreases with larger diameters at the pump inlet (PS2), while at the connection point (PS1) the pressure drop is almost independent of the service pipe diameter. This unexpected conclusion can be understood by considering that, for the same consumption flow rate, the larger the diameter of the service pipe, the smaller the flow velocity. Conversely, the reflection coefficient at the connection point will approach zero as the service pipe diameter is reduced. In other words, larger service pipe diameters will lead to smaller velocity changes when the pumps start and, consequently, to theoretically smaller pressure surges. However, the pressure drop generated by the pump start-up will be transmitted to the main pipeline more easily. Both effects act in opposite directions, each compensating for the other.

\section{Service pipe length}

For the numerical model used in this study, the length of the service pipe is measured from the pumps to the connection point of the service pipe and the main pipeline. This parameter clearly affects the transient magnitude, as it modifies the reflection time and the inertia of the water mass inside the pipe.

The longer the pipe, the longer the time it takes for the wave to travel from the pump to the connection point, and back again to the pump. A longer travelling time decreases the mitigating effect of the reflected pressure waves returning from the connection point. As shown in Fig. 4-g, the impact of this parameter is mainly seen on the suction side of the pump, while at the connection point the influence is almost negligible.

In addition, a longer service pipe increases the inertia of the fluid contained in it, making flow changes slower than with shorter pipes. A long service pipe can also have a great impact during the steady state operation of the system, as head losses due to friction may become significant. In

18

URL: http:/mc.manuscriptcentral.com/nurw Email: urbanwater@exeter.ac.uk 
networks with low pressure heads, the pressure on the suction side of the pump may not be sufficient to prevent the appearance of cavitation.

\section{Flow rate withdrawn from the network}

In order to analyze the effect of the velocity change in the service pipe for different diameters, the same 7 pumps modeled were used. For the simulations, the remaining parameters were those of the reference scenario. As shown in Fig. 7, there is a strong relationship between the velocity change in the service pipe $(\Delta v)$ and the maximum pressure drop on the suction side of the pumps $\Delta H_{\text {pump }}$ during the start-up. By means of this chart it is possible to estimate (for the building studied) the pressure head drop on the suction side of the pumps, provided that the final flow velocity under steady state conditions is known. Also, an analytical approximation of the maximum pressure drop can be obtained with the Michaud equation.

$$
\Delta H_{\text {pump }}=\Delta H_{0}-\frac{2 L \Delta v}{g \Delta t} \text { where } \Delta v<0
$$

where $L$ is the service pipe length, $\Delta t$ the time required by the pump to reach its nominal rotational speed and $\Delta H_{0}$ is the pressure drop at the building connection point (this last parameter can only be obtained by considering wave reflections and transmission within the public water network, as previously explained).

\section{SUMMARY AND CONCLUSIONS}

The study conducted presents a numerical analysis of water transients occurring during start-up when fixed-speed pumps installed inside residential buildings are connected directly to the public network. The results of the numerical analysis have been partially validated in a residential building in a major Spanish city.

The numerical simulations have identified two key parameters affecting the magnitude of the pressure surge: the actual flow rate capacity of the pump and the reflection coefficient at the connection point between the service pipe and the public main. The first parameter indicates the amount of water the pump is capable of extracting from the public network in a given installation.

19 
This is recorded at the intersection between the pump curve and the system curve. As a general rule, a larger flow capacity is associated with larger flow accelerations and, consequently, to larger underpressures. However, this parameter cannot be considered alone and it should always be associated with the amount of time required by the pump to reach its nominal rotational speed. For the same flow rate capacity, longer starting times will significantly decrease the magnitude of the pressure transient. This also explains why a variable-speed pump with a slow response does not produce significant water transients in the network. It can be safely installed as long as the public network has the required water transportation capacity under steady state conditions.

The second parameter, the reflection coefficient, indicates the ability of the public main to absorb the pressure wave created at the pumping station. A value of this coefficient close to one implies that very little of the pressure wave is transmitted to the public main. Therefore, pressure in the public network remains almost invariable without being affected by the operation of the pump. In such cases, fixed-speed pumps can be safely connected to the network. On the other hand, a reflection coefficient close to zero mean that the disturbance originated by the pump during the startup is mostly transmitted to the public mains. Under these circumstances, the connection of fixedspeed pumps can be extremely dangerous for the integrity of the network.

\section{Acknowledgement and disclaimer}

We would like to acknowledge the Empresa Metropolitana de Abastecimiento y Saneamiento de Aguas de Sevilla (EMASESA) for giving us the opportunity to modify and test the behavior of one of their buildings, and we would like to give especial thanks to Mr. Camilo Veiga, the head of water meter unit, for his technical support and endeavor.

\section{REFERENCES}

Basupi I., Kapelan Z. \& Butler D., 2014. Reducing life-cycle carbon footprint in the (re)design of water distribution systems using water demand management interventions, Urban Water Journal, 11(2), 91-107, DOI: 10.1080/1573062X.2012.750374. 
Bergant A., Tijsseling A., Vitkovsky J., Covas D., Simpson A. and Lambert M., 2008. Parameters affecting water-hammer wave attenuation, shape and timing-Part 1: Mathematical tools. Journal of Hydraulic Research. 46 (3), 373-381.

Cantor K.P., Lynch C.F., Hildesheim M.E., Dosemeci M., Lubin J., Alavanja J. and Craun G., 1998. Drinking water source and chlorination by-products I. Risk of bladder cancer. Epidemiology, 9-1, $21-28$.

Charalambous B., Charalambous S. and Ioannou I., 2007. Meter under-registration caused by ball valves in roof tanks. Water Loss 2007. Specialist Conference. International Water Association. ISBN: 978-973-7681-3. Bucarest, Rumanía.

Criminisi A., Fontanazza C.M., Freni G. and Loggia G.L., 2009. Evaluation of the apparent losses caused by water meter under-registration in intermittent water supply. Water Science and Technology, 60 (9), 2373-2382.

Clark R.M., Sivaganesan M., Selvakumar A. and Sethi V., 2002. Cost Models for Water Supply Distribution Systems. J. of Water Resources Planning and Management. 128 (5), 312-321.

Cobacho R., Arregui F., Cabrera E. and Cabrera E. Jr., 2008. Private water storage tanks: evaluating their inefficiencies. Water Practice \& Technology. IWA Publishing 2008. Doi:10.2166/wpt.2008.025.

Courtis B. J., West J.R. \& Bridgeman J., 2009. Chlorine demand-based predictive modeling of THM formation in water distribution networks. Urban Water Journal, 6 (6), 407-415, DOI: $10.1080 / 15730620903038461$.

Covas D., Stoianov I., Ramos H., Graham N., Maksimović C. and Butler D., 2004. Water hammer in pressurized polyethylene pipes: conceptual model and experimental analysis. Urban Water Journal, 1 (2), 177-197, DOI: 10.1080/15730620412331289977.

Hua F., West J.R., Barker R.A. and Forster C.F., 1999. Studies into chlorine decay in municipal water supplies. Water Research, 33 (12), 2735-2746.

21 URL: http:/mc.manuscriptcentral.com/nurw Email: urbanwater@exeter.ac.uk 
Kirmeyer G.J., Richards W., and Dery-Smith C., 1994. An assessment of water distribution systems and associated needs. Rep. American Water Work, Association Research Foundation, Denver.

Levesque S., Rodriguez M.J., Serodes J., Beaulieua C. and Proulx F., 2006. Effects of indoor drinking water handling on trihalomethanes and haloacetic acids. Water Research. 40-15. 29212930.

Mohamed H. and Gad A., 2011. Effect of Cold-Water Storage Cisterns on Drinking-Water Quality. J. Water Resour. Plann. Manage., 137(5), 448-455.

Nieuwenhuijsen M.J., Toledano M.B., Eaton N.E., Farell J. and Elliott P., 2000. Chlorination disinfection by products in water and their association with adverse reproductive outcomes: a review. Occup Environ Med. 57, 73-85.

Rizzo A. and Cilia J., 2005. Quantifying meter under-registration caused by the ball valves of roof tanks (for indirect plumbing systems). IWA Specialised Conference "Leakage 2005". Halifax, Canada. Conference Proceedings, p.106.

Rodriguez M.J., Sérodes J.B., Levallois P., 2004. Behavior of trihalomethanes and haloacetic acids in a drinking water distribution system. Water Research. 38-20. 4367-4382.

Rossman L.A., Clark R.M. and Grayman W.M., 1994. Modeling chlorine residuals in drinking-water distribution-systems. J. Environ. Eng., 120-4, 803-820.

Soriano J., 2012. Influencia de las instalaciones de suministro de agua de edificios sobre el comportamiento hidráulico en régimen permanente y transitorio de la red general de distribución Propuesta de una metodología para el análisis y gestión del parque de contadores de agua en un abastecimiento. Tesis Doctoral. Univ. Politécnica de Valencia, España.

Tamari S. and Ploquet J., 2012. Determination of leakage inside buildings with a roof tank, Urban Water Journal, 9:5, 287-303, DOI: 10.1080/1573062X.2012.660959.

Thorley A.R.D. and Chaudry A., 1996. Pump characteristics for transient flow analysis. Proc. 7th Int. Conf. on Pressure Surges, BHRA. Cranfield, Beds. (UK), pp. 461-475.

22

URL: http:/mc.manuscriptcentral.com/nurw Email: urbanwater@exeter.ac.uk 
1

2

3

4

5

6

7

8

9

10

11

12

13

14

15

16

17

18

19

20

21

22

23

24

25

26

27

28

29

30

31

32

33

34

35

36

37

38

39

40

41

42

43

44

45

46

47

48

49

50

51

52

53

54

55

56

57

58

59

60

Zhang Y., Zhu Z., Jin Y. and Cui B., 2012. Transient Characteristics of a centrifugal pump during starting period. J. Fluids Eng. (March 1982). 104- 1, 6-14. http://dx.doi.org/10.1115/1.3240859.

Villanueva C.M., Kogevinas M. and Grimalt J.O., 2003. Haloacetic acids and trihalomethanes in finished drinking waters from heterogeneous sources. Water Research. 37-4, 953-958. Doi:10.1016/S0043-1354(02)00411-6.

Wilo AG, 2007. Intelligent pumps for building automation systems, World Pumps, V. 2007- 490, 2632, ISSN 0262-1762, 10.1016/S0262-1762(07)70252-3. http://www.sciencedirect.com/science/article/pii/S0262176207702523).

Woolschlager J.E., Rittman B.E., and Pirious P., 2005. Water quality decay in distribution systems problems, causes and new modelling tools. Urban Water Journal, 2 (2), 69-79.

Wylie E.B. and Streeter V.L., 1993. Fluid Transients in Systems. Prentice Hall: Englewood Cliffs, New Jersey.

23

URL: http:/mc.manuscriptcentral.com/nurw Email: urbanwater@exeter.ac.uk 
List of figures:

Figure 1. Schematic of the hydraulic model used in ALLIEVI.

Figure 2. Water supply elements and configurations of the monitored building.

Figure 3. Measurements taken at PS1 and PS2; a) with a fixed-speed pump (and simulated pressure at PS2) b) with variable-speed pump.

Figure 4. Simulation of pressure drop originated for different system variables.

Figure 5. Water velocity and acceleration on the suction side of the pump.

Figure 6. Comparison of two parallel pumps ( $2 \mathrm{~s}$ delay) and one equivalent pump starting.

Figure 7. Summary of simulation results for the installation under study for several $\Delta \mathrm{v}$ and SP diameters.

24

URL: http:/mc.manuscriptcentral.com/nurw Email: urbanwater@exeter.ac.uk 


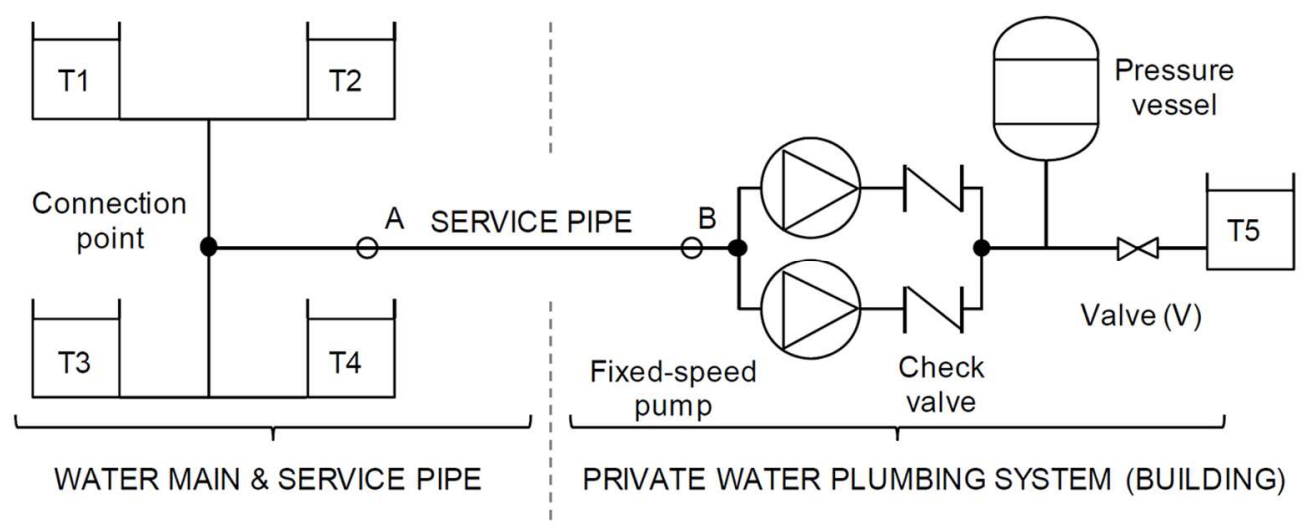

Figure 1. Schematic of the hydraulic model used in ALLIEVI $119 \times 48 \mathrm{~mm}(300 \times 300 \mathrm{DPI})$ 


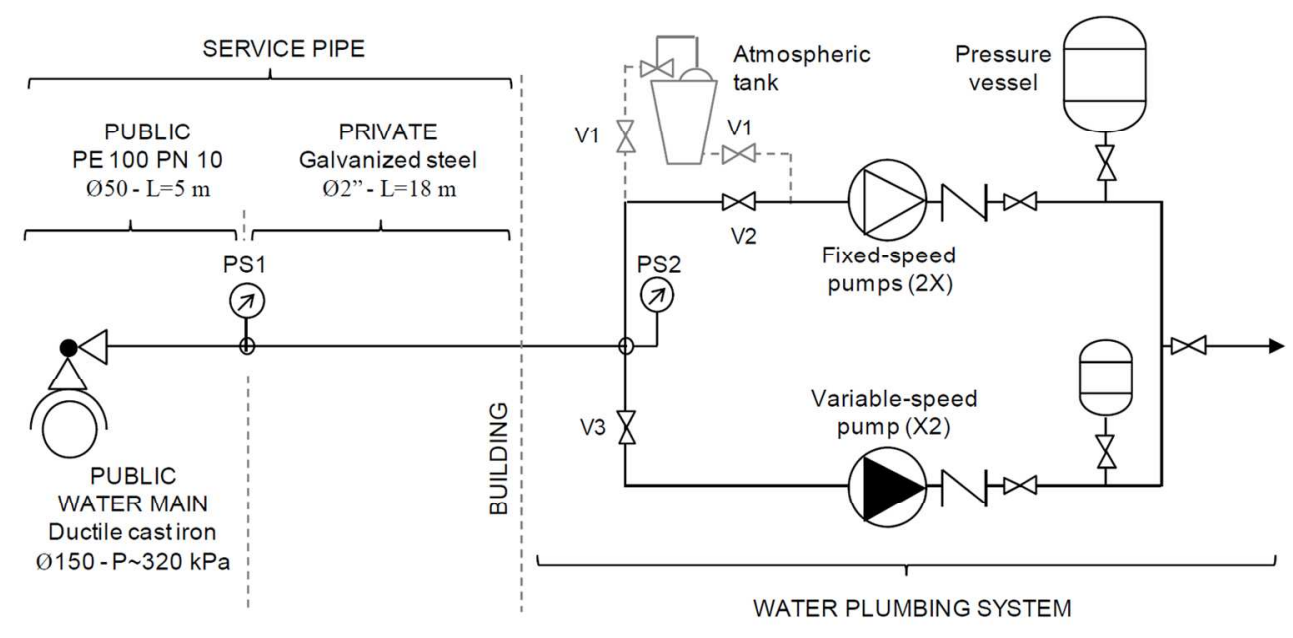

Figure 2. Water supply elements and configurations of the monitored building $140 \times 66 \mathrm{~mm}(300 \times 300 \mathrm{DPI})$

URL: http:/mc.manuscriptcentral.com/nurw Email: urbanwater@exeter.ac.uk 
a)

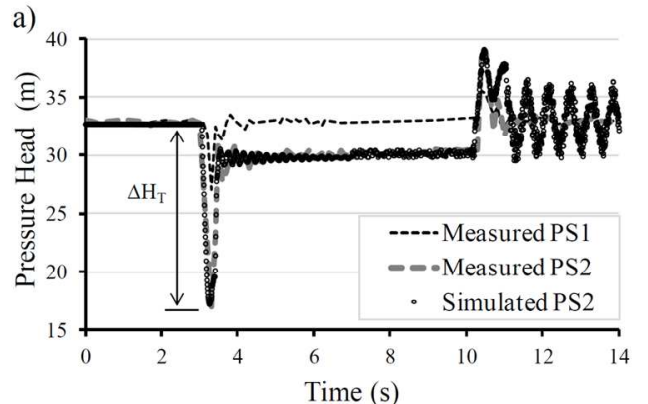

b)

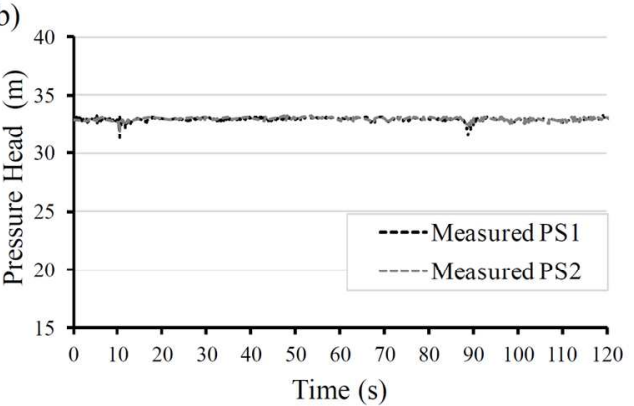

Figure 3. Measurements taken at PS1 and PS2; a) with a fixed-speed pump (and simulated pressure at PS2) b) with variable-speed pump. $160 \times 50 \mathrm{~mm}(300 \times 300 \mathrm{DPI})$ 


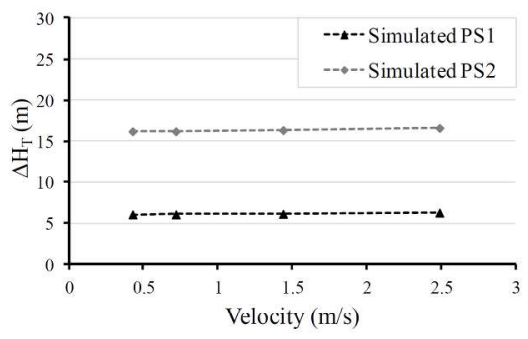

a) Influence of the flow-rate in the main pipe

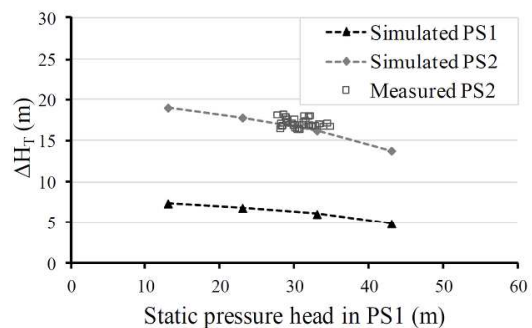

c) Influence of the pressure head at the connection point

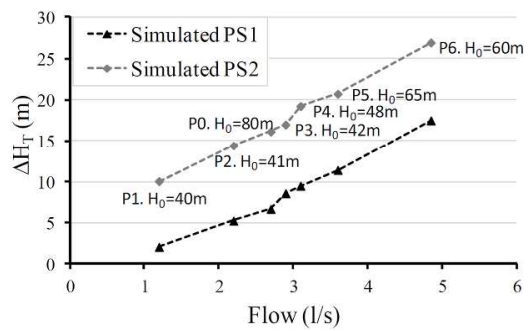

e) Influence of the pump flow-rate

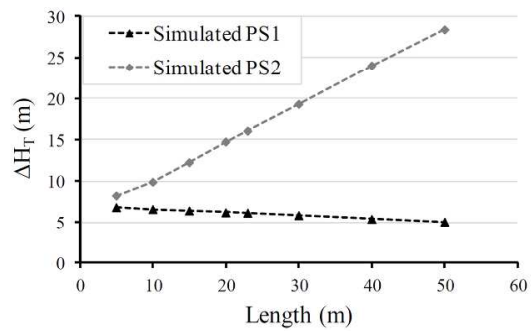

g) Service pipe length

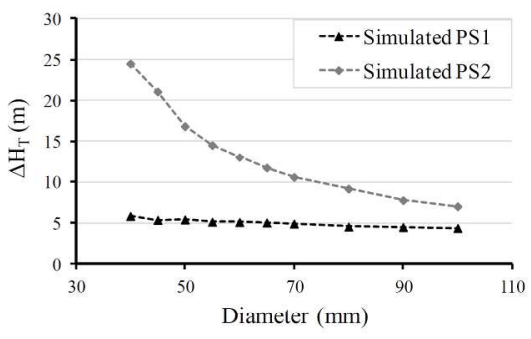

b) Influence of the water main diameter

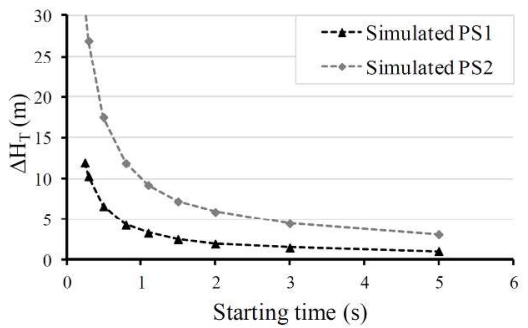

d) Influence of the pump starting time

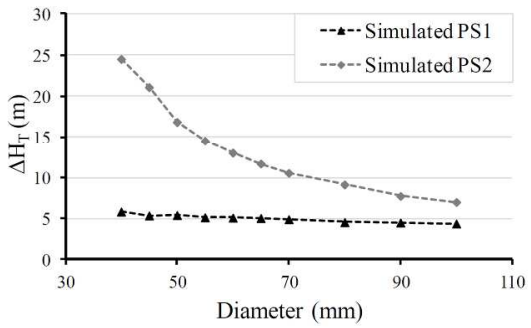

f) Influence of the service pipe diameter

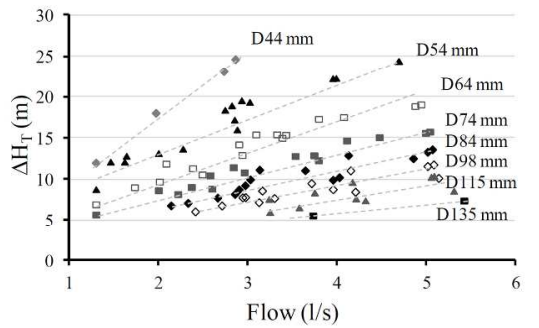

h) Influence of the pump flow-rate and SP diameter

Figure 4. Simulation of pressure drop originated for different system variables. $220 \times 304 \mathrm{~mm}(300 \times 300$ DPI)

URL: http:/mc.manuscriptcentral.com/nurw Email: urbanwater@exeter.ac.uk 


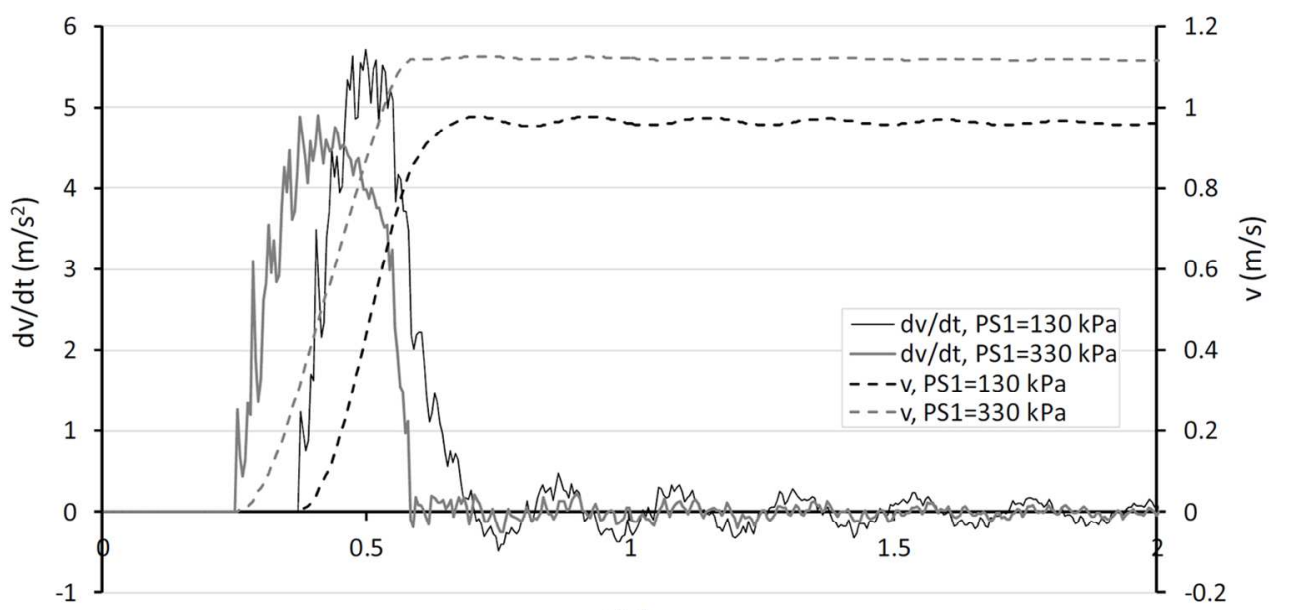

$t(s)$

Figure 5. Water velocity and acceleration on the suction side of the pump. $119 \times 59 \mathrm{~mm}(300 \times 300 \mathrm{DPI})$ 
Figure 6. Comparison of two parallel pumps ( $2 \mathrm{~s}$ delay) and one equivalent pump starting. $119 \times 62 \mathrm{~mm}(300 \times 300 \mathrm{DPI})$

URL: http:/mc.manuscriptcentral.com/nurw Email: urbanwater@exeter.ac.uk 
1

2

3

4

5

6

7

8

9

10

11

12

13

14

15

16

17

18

19

20

21

22

23

24

25

26

27

28

29

30

31

32

33

34

35

36

37

38

39

40

41

42

43

44

45

46

47

48

49

50

51

52

53

54

55

56

57

58

59

60

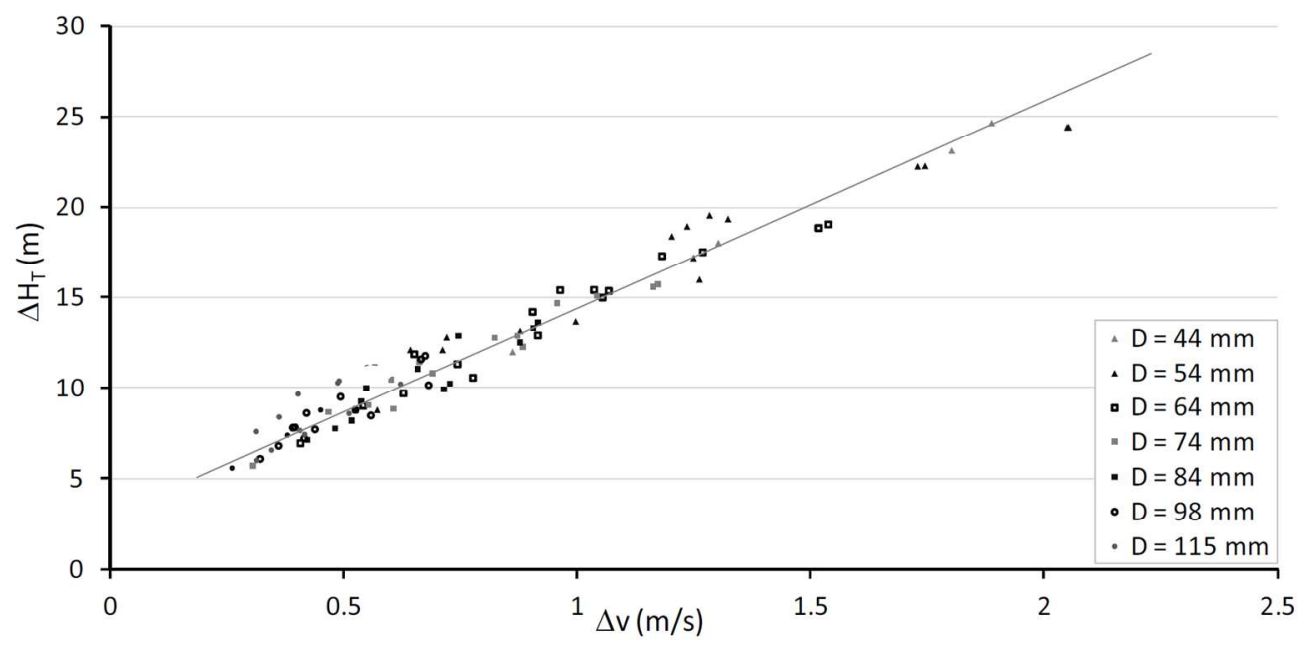

Figure 7. Summary of simulation results for the installation under study for several $\Delta \mathrm{V}$ and SP diameters. $160 \times 78 \mathrm{~mm}(300 \times 300 \mathrm{DPI})$ 